\title{
A Tangible User Interface as Interaction and Presentation Device to a Social Learning Software
}

\author{
Karin Leichtenstern, Elisabeth André, Eva Lösch \\ Multimedia Concepts and their Applications \\ University of Augsburg \\ Eichleitnerstrasse 30, 86135 Augsburg, Germany \\ \{leichtenstern, andre\}@informatik.uni-augsburg.de \\ eva.loesch@student.uni-augsburg.de
}

\author{
Matthias Kranz, Paul Holleis \\ Research Group Embedded Interaction \\ University of Munich \\ Amalienstrasse 17, 80333 Munich, Germany \\ \{matthias, paul\}@ hcilab.org
}

\begin{abstract}
This Birds-of-Feather paper presents an interesting use case for networked sensor nodes as part of a tangible user interface (TUI) in the context of a learning appliance. The software called "FearNot!" shows different bulling scenarios to children. At the end of each scenario, the spectators are asked for advice to the victim of the story how to cope with situations. Our objective is to investigate how different input devices and interaction techniques affect social learning. The Display Cube [11][8] is an appropriate sensor-node based user interface to present different options on each side of the cube and to select an option by turning the preferred option on the top and simply shaking the cube. The Display Cube is a networked sensing system that is connected via RF and UDP to the application logic. In this paper we describe both the technical implementation of the cube as well as information about our social learning software and the integration of the Display Cube as interaction and presentation device.
\end{abstract}

Index Terms - Tangible user interfaces (TUI), input device, interaction device, output device, presentation device, social learning, social learning.

\section{INTRODUCTION}

Children want to use technologies which support their curiosities, their love of repetition and their need for control as Druin points out [5]. Thus, in our opinion, new forms of interaction devices can increase children's involvement and willing to interact with computer software systems such as social learning software. Moreover, the interaction device should be intuitive to use and less obstructive. Therefore, there is a need for other input device than the classical interaction devices keyboard and mouse.

Humans are used to interact using their hands for interaction such as for making music, drawing, writing and so on. The question is why we are doing a step backward when interacting with computer system? Why not using the capabilities of the human hands for interactions? These issues are considered by tangible user interfaces (TUI) and in more general by perceptive user interfaces. We exploit the human capabilities of motor skills and imagination and use this as input to our sensing system. This allows for much more natural interactions. One goal of TUIs is increasing the efficiency of human-computer-interactions by optimizing input devices on human capabilities, not vice versa, requiring the human to learn a user interface. Ishii and Ullmer define the basic properties of TUIs in their grounding work of this user interface class [7]. Their intention is integrating our real world into the virtual world of bits and bytes so that we are feeling the virtual world as part of our real world. The gap between bits and atoms should be closed by making virtual information graspable. By manipulating this tangible representation the respective digital information can be changed. Small-scale sensor nodes prove to be a meaningful way of connecting physical objects via different networks to application interfaces.

We consider tangible user interacts (TUI) as exciting input device which enrich children's involvement with our social learning software. Thus, we decided to integrate the Display Cube [11][8] as interaction and presentation device in our social learning software. In the following, we describe some related work in this area. Afterwards we describe the technical implementation of the cube as well as the integration to new software FearNot!.

The contribution of this work is as follows: we ground our work on previous research on networked sensing systems, especially of tangible user interfaces including wireless sensor nodes. We present a new application scenario only enabled by the wireless sensing platforms connected via gateways to standard application interfaces. In this scenario, multiple networked sensor nodes incorporated in cubic tangible user interfaces, are used in a learning context. The interconnection of sensor nodes, not only in environmental monitoring, is an interesting field for research on networked sensor platforms to which we want to contribute to.

\section{RELATED WORK}

In this paper we used a tangible user interface in shape of a cube as input device. Sheridan [10] was one of the first studies the affordances of the cube as $3 \mathrm{D}$ object for humancomputer interaction.

The engagement and playfulness afforded by the cube has already been exploited in several occasions: besides the popular Rubrik's Cube, or the simple dices, HCI research has looked at the cube as user interface. Zhi Ying et al. [12] explore the application of a foldable 3D Cube interface, complemented with augmented reality technology, to the field of interactive storytelling. Camarata et al. [3] use cubes 
as physical blocks to navigate a data space in a virtual museum, thus exploiting the everyday understanding of three-dimensional spatial relationships.

Block et al. [2] used a tangible cube as input device for changing TV-channels. The cube contains capabilities to sense gravity. Thus, user's motion can be recognized and transmitted via a wireless communication interface to a screen. Block's cube is only a networked interaction device. The representation of the different options is shown on the screen as 3D graphical representation of the cube. Each displayed side of the cube shows a preview of the different TV streams. Figure 1 shows a user interacting with the prototype.

Another example of a cubic user interface is Laerhoven's Fair Dice [9]. It is an example of sensory augmentation of an object that perceives rolls and records what face it lands on.

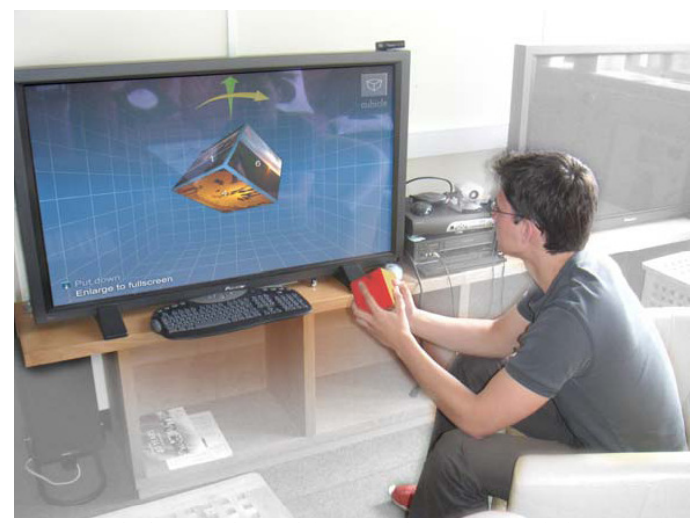

Figure 1: A user is interacting with the cube to control the TV-channels on the screen.

\section{DisPlay CUBE}

We used the Display Cube [11][8] because it supports as with a wireless device that provides interaction and presentation capabilities.

Figure 2 shows a picture of the included hardware and the Cube with its displays. For these capabilities the cube requires some technical hardware.

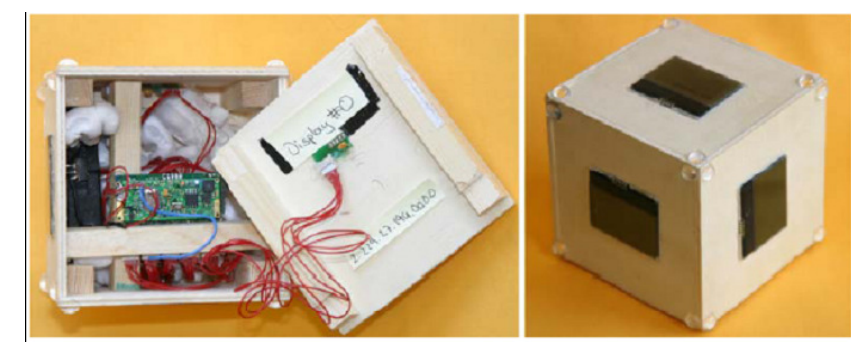

Figure 2: Picture of the Display Cube

The basic part of the Display Cube is a Particle Computer [4][1], a typical sensor node platform for ubiquitous computing. A Particle Computer supports whole technical requirements to build program-controlled devices on a small area. Moreover, base board functionality can be increased by augment the Particle Computer with additional sensors and add-on boards. The base board of a Particle Computer contains of a PIC 18F6720 micro controller, an antenna for sending and receiving radio frequency data and some sensors such as a accelerometer. The add-on board is connected via an I2C-connection to six displays. Moreover, the Display Cube contains two orthogonal arranged ADXL JE311 accelerometers which senses gravity and $G$ force. Figure 3 shows the complete hardware architecture of the Display Cube.

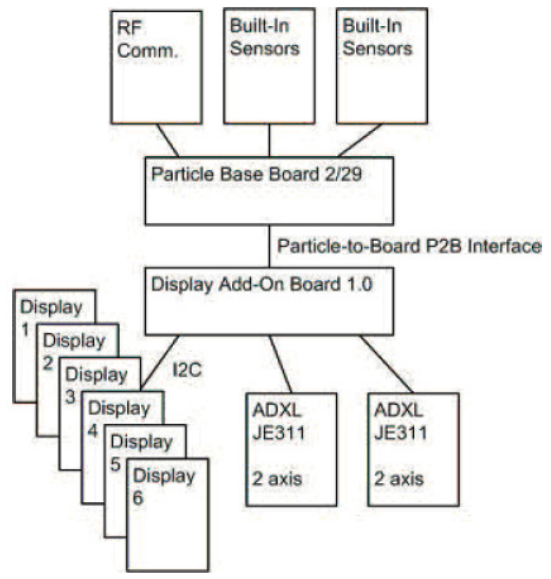

Figure 3: Hardware Architecture of the Display Cube

This architecture provides us with the required capabilities to act as an input and output media for our software. The input functionality bases on the accelerometers on the add-on board can recognize two required gestures to perform selections. The first gesture is the turning of the cube to know the current cube side on the top. The second gesture is shaking the cube to signal the selection of the proposal which is currently displayed on the top screen. For output functionalities the six displays are used. One displays shows victim's question and the other five displays show proposals to the victim such as "hit back". Finally the RF communication interface of the sensor node is required for two directions to receive the proposals from the computer display and two transmit the selected proposal back to the system.

\section{FEARNOT!}

Before describing the integration of the Display Cube to our social learning software, the current text based FearNot! version is introduced.

The EU-founded project E-Circus [6] investigates how social learning may be enhanced through interactive role play with virtual characters that establish empathetic relationships with the learners. It creates interactive stories in a virtual school with embodied conversational agents in the role of bullies, helpers, victims etc. The children run through various bullying episodes, interact with the virtual characters after each episode and provide advice to them. By taking on the perspective of the virtual characters, the children should come up with a better understanding of how to cope with 
such situations. We suppose arranging interactions using tangible user interfaces such as the Display Cube increases social interactions between children and foster social skills.

Fig. 4 shows a screenshot of the software FearNot! In which a bulling situation is shown to children.

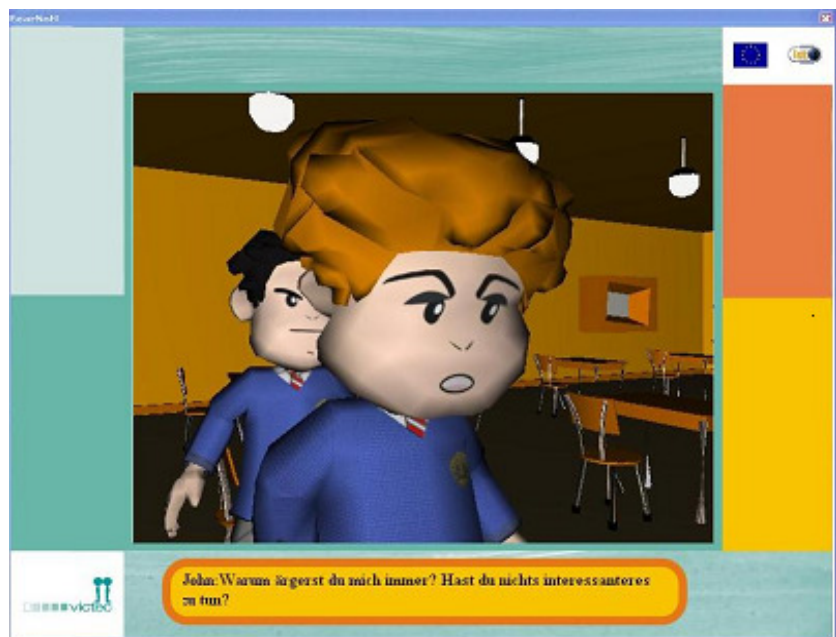

Fig. 4: Screenshot of a Bulling Scenario

After the bullying situation, the victim asks the spectator for a proposal how to cope with this situation. The children can type in a proposal in current FearNot! version as displayed in Fig. 5.

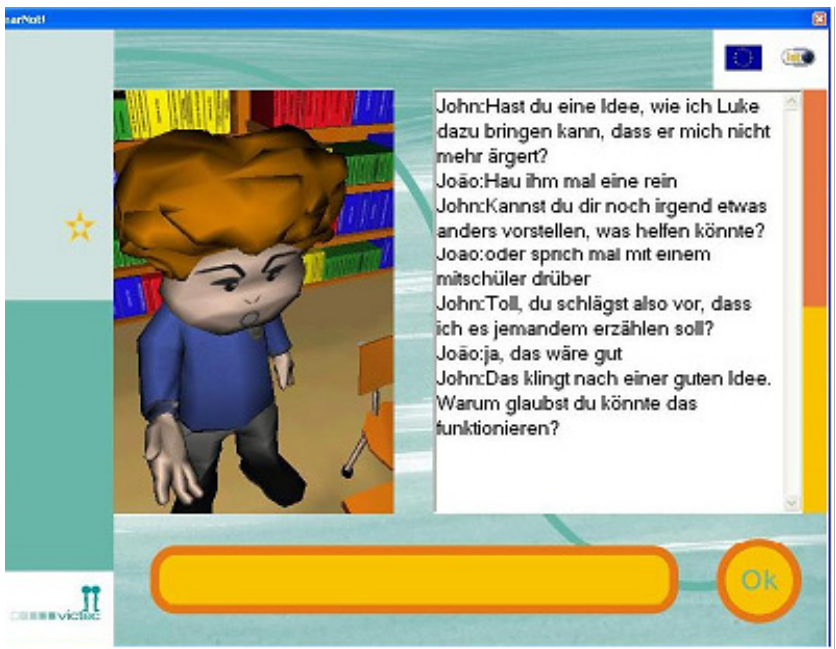

Fig. 5: The victim is asking the spectators for a proposal how to cope with the current situation.

The next social learning software in e-Circus is called ORIENT. It does not consider bullying anymore but integration issues of foreigners. For this software other interaction techniques should be integrated. It is planned to combine a verbal and non-verbal interaction for spectators. For non-verbal interaction, devices are currently searched which enables representations of information and interaction with the software. To verify some devices on these requirements, we integrate several interaction devices to FearNot!. We consider tangible user interfaces as appropriate for us. In our point of view the Display Cube fulfills lots of the requirements. Thus, we integrated the Display Cube in the current FearNot! version and called this version FearNot!Extended.

\section{FEARNOT!EXTENDED: INTEGRATION OF THE DISPLAY CUBE TO FEARNOT!}

We used our current text based FearNot! version and integrated the Display Cube. Multiple Display Cubes can be used in a multiuser scenario. The following section describes the architecture and interaction style for FearNot!Extended.

Fig. 6 shows the architecture of our current system which consists of two main components. On the left side, there is the desktop PC which shows the learning software FearNot!. On the right side, the Display Cube which is used as interaction and presentation device for non-verbal interaction with our software, is depicted.

The desktop PC requires a ParticleComm API to use a USB-Bridge (Fig. 7). Using the RF-to-UDP Xbridge or USBBridge, a communication between a desktop PC and the Display Cube is possible. Thus, FearNot! is able to transmit the proposals once the spectator of the scenario has to give the victim an advice via a local and then via a wireless sensor network.

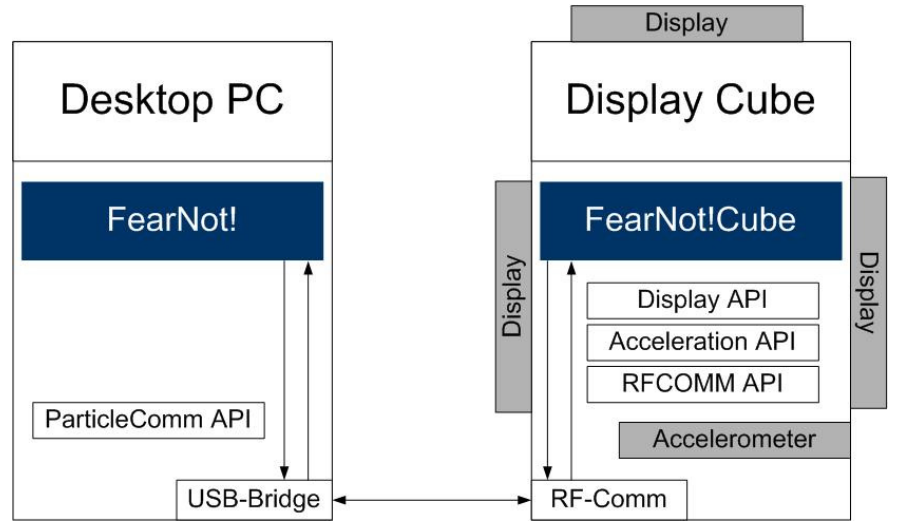

Figure 6: The architecture of FearNot!Extended and the integration of the Display Cube is depicted.

The Display Cube contains an application called FearNot!Cube which uses several APIs. The application requires an API to address the different displays and to rewrite them. Moreover, there is a need to read and interpret sensor data from the accelerometer which is done by the Acceleration API. Finally, the application requires access to the RF-Comm interfaces. Therefore, the RFCOMM API is used to receive and transmit data.

Fig. 7 illustrates a typical interaction process. First FearNot!Extended is started on the desktop PC. Then a scenario is shown which ends with ask for advice. At this point, a Display Cube is required.

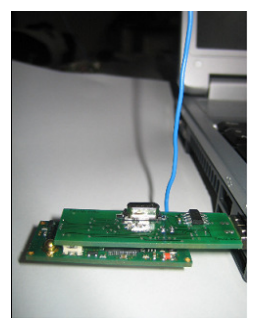


Fig. 7: An USBBridge to enable a desktop PC a RF communication to the Display Cube.

The desktop PC transmits via its USBBridge data to the Display Cube. This data are received and interpreted by the Display Cube. As consequence, the Display Cube shows the respective proposals on the different cube sides.

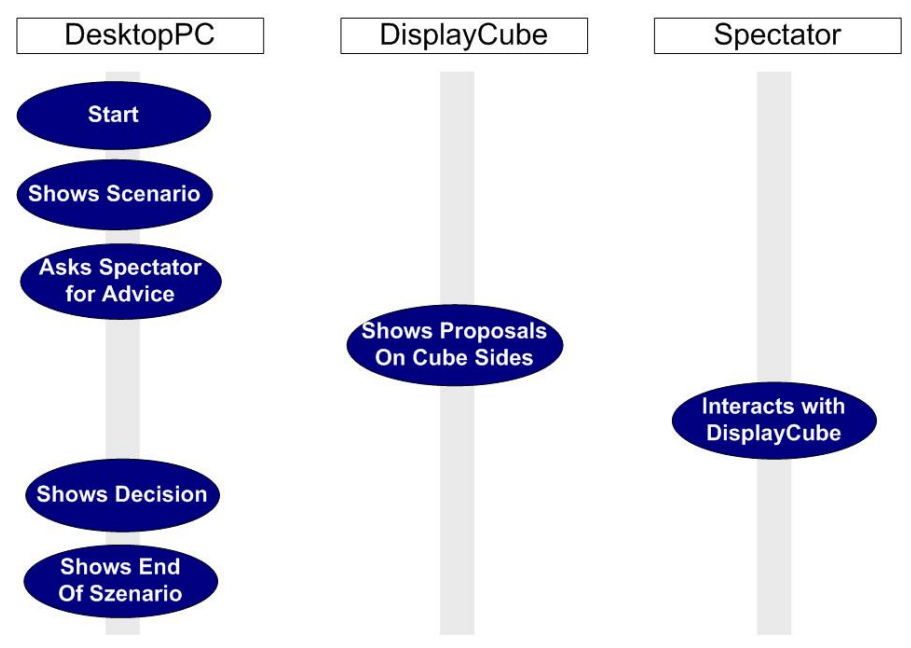

Fig. 8: A typical Interaction Process in the FearNot!Extended system.

Now the user can interact with the Display Cube. The spectator is turning the cube to see all different options which is shown in Fig. 8. Once the user has decided which advice to give, he is simply shaking the cube to signal the selection. This shaking is recognized by FearNot!Cube and leads to an transmission back to the desktop PC. The desktop PC displays the end of the scenario which shows the spectator a consequence on his proposals.

\section{CONCLUSION}

In this paper we used a tangible user interface called Display Cube, based on a common sensor network platform, as interaction presentation device for social learning software. First we described the technical implementation of the Display Cube. Afterwards we introduced our software FearNot! and FearNot!Extended. We described the integration of the TUI to our software via RF and UDP based communication networks. We showed that TUIs can replace classical input devices as a mouse and a keyboard. This has been verified in an evaluation with children in an age of 13 up to 14. We found out that they could use the Display Cube as input device.

We consider the Display Cube as appropriate device because of its two modalities. The spectator is forced to switch their gaze from the display to the Display Cube and back. Moreover, they are forced to use their hands to turn the cube to see each option and to perform the shaking.
We showcased the potential of small scale sensor nodes using different physical networks as input devices for desktop based learning software. We are looking to discuss the work presented in this paper in the Birds-of-Feather session with the conference participants.

\section{REFERENCES}

[1] Beigl, M., Krohn, A., Zimmer, T., Decker, C. and Robinson, P.: AwareCon: Situation Aware Context Communication, in Ubicomp 2003, Seattle, USA, 2003.

[2] Block, F., Schmidt, A., Villar, N. and Gellersen, H.W.:Towards a Playful User Interface for Home Entertainment Systems. European Symposium on Ambient Intelligence (EUSAI 2004). Springer LNCS 3295, pp. 207-217, Springer, 2004.

[3] Camarata, K., Yi-Luen Do, E., Johnson, B., Gross, M. D.: Navigational Blocks: Navigating Information Space with Tangible Media. Int. Conf. on Intelligent User Interfaces, ACM Press, 2002.

[4] Decker, C., Krohn, A., Beigl, M., and Zimmer, T.: The particle computer system, in Proceedings of the ACM/IEEE Fourth International Conference on Information Processing in Sensor Networks, April 2005.

[5] Druin, A.: The Design of Children's Technology. Moran Kaufmann Publishers. 1999.

[6] E-Circus: http://www.e-circus.org/.

[7] Ishii, H., and Ullmer, B.: Tangible Bits: Towards Seamless Interfaces between People, Bits and Atoms. In: Proceedings of CHI'97, 1997. pp. 22-27.

[8] Kranz, M., Holleis, P. and Schmidt A.: A Display Cube as a Tangible User Interface. In: Adjunct Proceedings of UbiComp, September 2005.

[9] Laerhoven, K.V. Gellersen, H.-W.: T Fair Dice: A Tilt and Motion-Aware Cube with a Conscience. IWSAWC, p. 66, 2006

[10] Sheridan, J., Short, B., Laerhoven, K.V., Villar, N. and Kortuem, G.: Exploring cube affordance: Towards a classification of non-verbal dynamics of physical interfaces for wearable computing. In Proceedings of the IEE Eurowearable 2003. IEE Press, 2003, pp. 113 - 118.

[11] Terrenghi, L., Kranz, M., Holleis, P., Schmidt, A.: A Cube to Learn: a Tangible User Interface for the Design of a Learning Appliance. 3AD: Third International Conference on Appliance Design. Laboratories, Bristol, UK, June 2005. pp. 82-86.

[12]Zhi Ying Zhou, Pan Jiun Horng, Adrian David Cheok, Yu Li: 3D Story Cube: an Interactive Tangible User Interface for Storytelling with 3D Graphics and Audio. Personal Ubiquitous Computing, 8(5), 2004. 